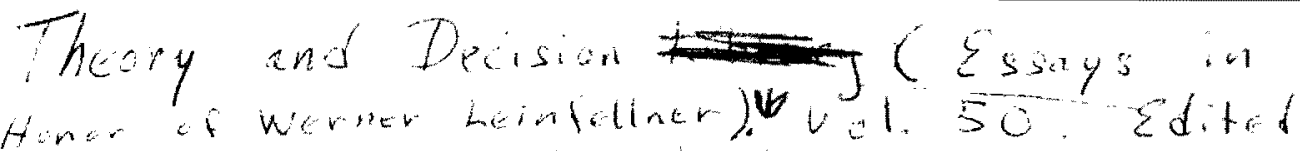

$$
\begin{aligned}
& \text { b. Hal Berghel and Gerated Eberlein } 7 \times 8 \text { D Redel } \\
& \text { PHILIP HUGLY AND CHARLES SAYWARD }
\end{aligned}
$$

\title{
WHY SUBSTITUTIONAL QUANTIFICATION \\ DOES NOT EXPRESS EXISTENCE
}

Fundamental to Quine's philosophy of Jogic is the thesis that

(Q) Substitutional quantification does not express existence.

We here consider the content of this claim and the reasons for thinking it true.

1. On our usage a sentence is an existential quantification only if it is a sentence in the standard assertive use of which speakers commit themselves to the existence of things. Existential quantifications are formed by a variable binding operation. Further, these sentences play a certain familiar role in inference. But not every sentence satisfying these conditions is an existential quantification, as is shown by the possibility of forming sentences by binding variables the substitutends for which are, say, parentheses.

Reflecting on such punctuational quantifications, it is natural to explain their evident lack of existential content through the observation that sentential positions accessible to parentheses are not referential. And this in turn suggests that it is sufficient for existential content in a quantification that the position of its bound variable be referential.

But what makes a position referential? Here it is natural to connect the idea of referential position with the idea of accessibility to an individual constant.

2. That a quantification is existential only if the position of its bound variable is accessible to an individual constant we are ready to grant. But is this condition also sufficient? According to Quine it is not. And we agree. For $(E a) A$ asserts existence only if it is not substitutional and it can be substitutional even if individual constants are among the substitutends for $a$.

But what is it for a quantification to be substitutional?

3. Our explanations will employ some standard terms. First, by $L$ we mean a first order quantificational system and by $M$ a model for $L$. $L$ is

\section{7}

Gerald Eberlein and Hal Berghel (Eds.): Theory and Decision, 67-75.

(C) 1988 by D. Reidel Publishing Company. 
a language, represented by a pair $\langle L, M\rangle$. Sentence $S$ of $\mathbf{L}$ is true in $\mathbf{L}$ just in case $S$ is true in $M$. Truth in $M$ can be construed either in terms of an objectual definition of truth (ODT) or in terms of a substitutional definition of truth (SDT). We say that the SDT for $\mathbf{L}$ is deviant just in case it and the ODT for $\mathbf{L}$ differently distinguish truths from non-truths, as will be the case if some degree-one formula $A$ is satisfied by at least one domain element but by no domain element assigned any individual constant. Finally, we consider the following definition:

(1) Quantification is $\mathbf{L}$ in substitutional just in case the SDT for $\mathbf{L}$ is non-deviant.

Though this seems to be on the right track, there is reason to think it too weak to fully capture the intended content of $(Q)$. For consider the case of a pair of languages $\mathbf{L}$ and $\mathbf{L}^{\prime}$ where the SDT for $\mathbf{L}$ misses nondeviance by just one unassigned domain element and $\mathbf{L}^{\prime}$ differs from $\mathbf{L}$ just in having an additional individual constant assigned that element. By $(Q)$, no quantification of $L^{\prime}$ expresses existence.

Now, are we willing to suppose that though the quantifications of $\mathbf{L}^{\prime}$ do not express existence, the quantifications of $\mathbf{L}$ do express existence? That just isn't plausible. The minimal move of adding or dropping an assigned individual constant cannot make for the difference between quantifications which do and don't serve for the assertion of existence.

Evidently, we need a revision of (1) which will deem the quantifications of $\mathbf{L}$ as well as those of $\mathbf{L}^{\prime}$ non-existential. We shall call a language $\mathbf{L}^{\prime}=\left\langle L^{\prime}, M^{\prime}\right\rangle$ a name extension of $\mathbf{L}$ just in case $L^{\prime}$ results from $L$ by adding at most countably many individual constants to $L$, and $M^{\prime}$ differs from $M$ just by determining denotations for those new individual constants. As our new definition we consider.

(2) Quantification in $\mathbf{L}$ is substitutional just in case there is some name extension $\mathbf{L}^{\prime}$ of $\mathbf{L}$ the SDT for which is non-deviant.

Interpreting (Q) by (2) seems just right - for the distinction it draws between substitutional and non-substitutional quantification seems to coincide with the intended distinction between existential and nonexistential quantifications.

4. But in fact the situation is just the opposite, for by (2) all first order languages are substitutional, whereas it seems clear that not all first 
order languages lack existential quantifications. That (2) rules all first order languages substitutional can be seen by noting that

For any $\mathbf{L}$, there is a countable name extension $\mathbf{L}^{\prime}$ of $\mathbf{L}$ the SDT for which is non-deviant. ${ }^{1}$

Our attempt 10 employ the notion of substitutionality to draw a line between existential and non-existential quantifications thus seems to end in failure. On the other hand, there still seems to be something right in (Q). But what is it that seems right in (Q)?

5. It will prove useful at this point to reflect on the natural basis we have for supposing that substitutional quantification, in the simple sense of (1), is non-existential.

To come to this basis, first reflect again on the idea of referential position. Though it was natural to suppose that accessibility to individual constants marks a position as referential, a language may provide such positions but not actually include such terms. What, then, marks a language as one providing positions accessible to individual constants? It will not be that the language employs variables - for there are, e.g., sentential variables marking positions which are accessible only to formulas, not to terms. Rather, it will be that the language employs predicates. And though predicates provide positions accessible to individual constants, the more fundamental point is that predicates provide referential positions. The entirely plausible idea, central to Quine's philosophy of logic, is that just as a term is a term of reference by being accessible to positions determined by predicates, so also a variable has values by being accessible to such positions. It is the notion true of, central to the semantics of predicates, which is our primary referential notion.

Next, reflect on our use of individual constants in a first order language. That use is primarily to form elementary sentences. If all truth-values in the language are a function of the truth-values of these elementary sentences, then the language is straightforwardly substitutional. But also, in that case, there is no need to regard the expressions which combine with the individual constants to form elementary sentences as predicates, i.e., as expressions true of things. Thus, straightforwardly substitutional languages will be ones the quantifications of which do not serve to assert the existence of things for the reason that devices of reference are entirely lacking in such languages. 
6. Now when we say the "there is no need" to regard any expressions of $L$ as predicates and then infer that there are no such expressions in $L$ we have in mind a certain fundamental principle of semantical theorizing, namely

(T) A language has no semantical properties beyond those required for devising an adequate semantics for it.

It is a principle in the spirit of Occam's Razor. We illustrate its plausibility by means of a simple example.

Consider the simple language $L^{*}$ with just " $P 1$ ", " $P 2$ ", and " $P 3$ " as atomic sentences and further sentences by prefixing " $N$ " to two occurrences of sentences or prefixing " $(E a)$ ", " $(E b)$ ", etc. to the expressions which result from replacing occurrences of "l", " 2 ", or " 3 " in sentences by occurrences of " $a$ ", " $b$ ", etc. We will suppose that each atomic sentence is an environmental sentence used to assert a weather condition on each occasion of its use. Thus, e.g., we may suppose that an utterance of " $P 1$ " on a given occasion yields a truth on that occasion just in case the environment of the utterance is rainy. We further suppose that " $N$ " is for joint denial and that, e.g., "( $E a) P a "$ yields a truth on an occasion of utterance just in case it is rainy, sunny, or windy in the environment of that utterance.

In effect, the three atomic sentences are one-word sentences of the sort a very young child might use. The truth-predicate in a semantical account of $\mathbf{L}^{*}$ would be something like this,

$s$ is true at $p$ at $t$

where " $s$ ", " $p$ ", and " $t$ " are variables for sentences, places, and times. The full semantics would run roughly as follows:

(1) "P1" is true at $p$ at $t$ iff it is rainy at $p$ at $t$.

"

$"$

(4) For any sentence $N s r, N s r$ is true at $p$ at $t$ iff neither $s$ nor $r$ is true at $p$ at $t$.

(5) For any sentence $(E a) A,(E a) A$ is true at $p$ at $t$ iff at least one of $A a / 1, A a / 2$ and $A a / 3$ is true at $p$ at $t$.

On this semantics for $\mathbf{L}^{*}$ the variables of $\mathbf{L}^{*}$ are not assigned values. But other semantical accounts for $\mathbf{L}^{*}$ are available. For example, let DEN be a function assigning 1,2 , and 3 to " 1 ", " 2 ", and " 3 " and the 
nth natural number to the $n$th variable of $\mathbf{L}^{*}$. A semantics for $\mathbf{L}^{*}$ could then run roughly as follows:

(1) $n$ satisfies " $P 1$ " at $p$ at $t$ iff it is rainy at $p$ at $t$.

(2) $\quad n$ satisfies $P a$ at $p$ at $/$ iff $\operatorname{DEN}(a)=n$ and it is rainy at $p$ at $t$.

"

"I

(7) $\quad n$ satisfies $N s r$ at $p$ at $t$ iff $n$ satisfies neither $s$ nor $r$ at $p$ at $t$.

(8) $\quad n$ satisfies $(E a) A$ at $p$ at $t$ iff for some $m, m$ satisfies $A a / b$ at $p$ at $t$ for the first variable $b$ new to $A$.

Finally, for any sentence $S$ of $\mathbf{L}^{*}, S$ is true at $p$ at $t$ iff for each $n, n$ satisfies $S$ at $p$ at $t$.

It would be easy to show that these two semantical accounts of $\mathrm{L}^{*}$ are equivalent in the sense that they effect the same classifications of both truths and logical truths of $\mathbf{L}^{*}$.

Which account "correctly" represents $L^{*}$ ? We would here invoke Occam's Razor and opt for the simpler account. The apparatus of numbers plays no essential role in the semantical account of $\mathbf{L}^{*}$. It is like the wheel on Wittgenstein's machine - it turns without turning anything else.

What seems plain is that $\mathbf{L}^{*}$ is a non-ontological language. Its atomic sentences are, in Strawson's apt term, feature placing sentences. The remaining sentences are just molecular compounds of these feature placing sentences and quantifications abbreviating certain of those molecular compounds. Our simple semantics is true to the evident character of this language. The complex semantics isn't.

On the basis of principle $(T)$ and the fact that the simpler account of $\mathbf{L}^{*}$ is an adequate semantics for $\mathbf{L}^{*}$ which treats no expression of $\mathbf{L}^{*}$ as an $n$-ary predicate true of $n$-tuples of objects, we conclude that $\mathbf{L}^{*}$ has no $n$-ary predicates. In that case the positions accessible to variable are non-referential. Thus, the quantifications are non-existential, i.e., the quantifications of $\mathbf{L}^{*}$ lack existential import.

A substitutional semantics is based on the idea that all truth is a function of some truth. The notion true of plays no role in such a semantics. So if a substitutional semantics is adequate for a language $\mathbf{L}$ then, by principle (T), L's sentences lack referential positions. The atomic sentences function as one word sentences whose subsentential 
parts play no semantical role. But we agreed that a quantification is an existential quantification only if the positions of its bound variable are referential. We conclude that if a substitutional semantics is an adequate semantics for $\mathbf{L}$ no quantification in $\mathbf{L}$ is existential.

7. The argument just given concludes

(a) If the SDT for a language is adequate none of the language's quantifications are existential.

But under what conditions is the SDT adequate? One possible answer is

(b) The SDT for a language is adequate if it is non-deviant,

from which we may infer

(c) If the SDT for a language is non-deviant then none of the language's quantifications are existential.

Does this conclusion capture what truth there is in $(\mathrm{Q})$ ?

Imagine a group of speakers employing a first order language in talking about presently existing cows. As individual constants, they use the ordinary numerals. They adopt the following naming procedure: Explore for cows. Name each cow in order of discovery by the corresponding numeral. Thus the first cow discovered is named by "1", and so on. It may be that proceeding in this way they will have named all the cows. But no person could ever know that, certainly not if we are speaking of all regions of space. The people who speak this language do not automatically assent to a universal generalization $(a) A$ even if they are certain of each $A a / t$. Nor do they automatically reject $(E a) A$ even when they are certain each $A a / t$ is false. The objection is that even if the SDT for the language were to turn out to be non-deviant, a substitutional semantics would not correctly represent the language of these speakers. Such a semantics would not be in accord with the predominate usage of the language. The speakers of the language do not use their quantifications substitutionally.

We think this example shows that (b) above is false. Non-deviance does not guarantee adequacy. Further conditions are required.

Moreover, the question "Is the SDT for $\mathbf{L}$ adequate?" is misstated. We should ask instead, "Is the SDT for $\mathbf{L}$ adequate relative to group $G$ of speakers of $\mathbf{L}$ ?"

Imagine a world much like our own except that model theory has not 
come into existence. In this world there are two groups of mathematicians who speak the same language of arithmetic. Suppose the Gödel result that decidable axiom sets suitable for arithmetic are incomplete if consistent is known by the two groups. That is, they have this syntactical knowledge: if a set $\nabla$ of axioms is decidable and, e.g.", $" 0=1$ " is not derivable from $\nabla$, then, for some sentence $\phi$ of arithmetic, neither $\phi$ nor $7 \phi$ is derivable from $\nabla$. They reflect on the undecidable sentence which is of the form $(x) A(x)$. They note that $A(0), A(1), A(2), \ldots$ are each provable from a suitable axiom set $\nabla$ which both groups accept. Suppose that at this point the reactions of the two groups diverge. One group, the $S$ s, willingly accept $(x) A(x)$ because each of its instances is provable from axioms they accept. They remain quite clear, however, that no proof exists for $(x) A(x)$ from these axioms. The other group, the $T$ s, accept neither $(x) A(x)$ nor its negation on the grounds that no proof exists for these propositions.

The $S$ s bring epistemic closure to their quantifications. According to their practice, one is warranted in asserting any universal quantification if one is warranted in asserting each instance; similarly one is warranted in asserting $(E a) A$ only if some $A a / t$ is warrantedly assertable. But the $T s$ do not bring such epistemic closure to their quantifications. It is in this difference of practice that we must lind the difference between having and lacking existential import.

From a formal semantical point of view the SDT is non-deviant for $L$, whether as spoken by the Ss or $T \mathrm{~s}$. But the SDT is adequate for this language of arithmetic only relative to the $S$ s; it is not adequate for the same language relative to the $T \mathrm{~s}$.

Generally,

The SDT for a language $\mathbf{L}$ is adequate relative to a group $G$ of speakers of $\mathbf{L}$ if (i) the SDT is non-deviant for $\mathbf{L}$ and (ii) the predominant practice in $G$ is to bring epistemic closure to L's quantifications.

We slaall also stand by the argument in Section 6 as establishing that no quantification in $\mathbf{L}$ is existential relative to group $G$ of speakers of $\mathbf{L}$ if the SDT for $\mathbf{L}$ relative to $G$ is adequate. Combining the two claims we conclude that a good part of what truth there is in $(Q)$ comes to this: For any language $\mathbf{L}$ and group $G$ of speakers of $\mathbf{L}$, none of $\mathbf{L}$ 's quantifications are existential relative to $G$ if (i) the SDT for $\mathbf{L}$ is nondeviant and (ii) the predominate praclice in $G$ is to bring epistemic closure to L's quantifications. 
8. We have agreed with Quine on the following point: some but not all quantificational assertions (assertions made in the use of quantificational sentences) are existential assertions (assertions of the existence or nonexistence of things). The question we have addressed is that of what it is which distinguishes existential quantificational assertions from nonexistential quantificational assertions.

A basic thesis of much work on existential commitment - Quine's included - is that this distinction coincides with the distinction between objectual quantification and substitutional quantification, and that this latter distinction is specifiable in formal terms.

Since existential and non-existential quantificational assertions can involve syntactically identical sentences of syntactically identical languages, there is no syntactic difference between existential and nonexistential quantificational assertions. Thus, if this distinction can be formally drawn, it will have to be drawn in semantical terms.

We then argued that the distinction cannot be captured in semantical terms. Our basic line of thought was this: we agree that the only distinction of formal semantics which is a plausible candidate for distinguishing between existential and nonexistential quantification is the distinction between objectual and substitutional quantification, but argue that this distinction does not coincide with the desired one.

Since the desired distinction cannot be captured in syntactical or semantical terms, we conclude that the distinction is due to some aspect of our use of language which can vary though the language used is kept syntactically and semantically constant.

We conjectured that the feature of usage in terms of which the distinction can be captured is what we have called bringing speech to epistemic closure. Were this conjecture correct, whether the speakers of some language (with a fixed syntactic structure and semantical metatheory) made existential assertions in their use of the quantificational sentences of that language would depend on their preparedness to countenance counterexamples. If so prepared, their quantificational assertions will be existential, and otherwise not.

The examples we gave in support of this conjecture fall short of establishing it. So it remains a conjecture, but one which we hope merits further research.

University of Nebraska-Lincoln 


\section{NOTE}

1 Hugly, Philip and Sayward, Charles: 1982. 'Indenumerability and Substitutional Quantification', Notre Dame Journal of Formal Logic 23, 358-366. 